\title{
$\begin{array}{ll}\text { Research Square } & \text { Preprints are preliminary reports that have not undergone peer review. } \\ \text { They should not be considered conclusive, used to inform clinical practice, }\end{array}$ or referenced by the media as validated information. \\ Tilt illumination for structured illumination imaging
}

\section{Xin Jin}

Harbin Institute of Technology

Xuemei Ding

Harbin Institute of Technology

Jiubin Tan

Harbin Institute of Technology

\section{Cheng Shen}

California Institute of Technology

\section{Xuyang Zhou}

Harbin Institute of Technology

Shutian Liu

Harbin Institute of Technology

Zhengjun Liu ( $\boldsymbol{\nabla}$ zjliu@hit.edu.cn )

Harbin Institute of Technology https://orcid.org/0000-0003-2886-8496

\section{Research Article}

Keywords: Tilt illumination, Structured illumination, Super-resolution imaging

Posted Date: February 19th, 2021

DOl: https://doi.org/10.21203/rs.3.rs-181084/v1

License: (c) (1) This work is licensed under a Creative Commons Attribution 4.0 International License.

Read Full License

Version of Record: A version of this preprint was published at Optical and Quantum Electronics on August 14th, 2021. See the published version at https://doi.org/10.1007/s11082-021-03174-6. 


\title{
Tilt illumination for structured illumination imaging
}

\author{
Xin Jin $^{1,2}$ • Xuemin Ding ${ }^{1,2}$ • Jiubin \\ $\operatorname{Tan}^{1,2}$. Cheng Shen ${ }^{3}$ - Xuyang Zhou ${ }^{1,2}$. \\ Shutian Liu $^{4}$. Zhengjun Liu ${ }^{1,2, *}$
}

Received: date / Accepted: date

\begin{abstract}
To achieve super-resolution imaging, the information in higher frequency of the observed sample is collected by illuminating with a structure beam for a limited optical transfer function (OTF). In this paper, tilt illumination mode is introduced to structured illumination microscopy (SIM) for enhancing lateral resolution. More sample spectrum more than traditional SIM, can be obtained by detector. Thus, SIM with tilt illumination can be improved at the aspect of lateral imaging resolution.
\end{abstract}

Keywords Tilt illumination · Structured illumination · Super-resolution imaging

\section{Introduction}

Structured illumination microscopy (SIM) is a tool of super-resolution imaging. In 2000, Gustafsson optimized the generation mode of sinusoidal structured light for illumination [1], which successfully has a double resolution in some imaging systems $[2,3,4,5,6]$. SIM has been widely used in optical sectioning, phase imaging, and 3D surface measurement [7]. As an expanded case, digital grating was introduced into virtual structured detection $[8,9,10]$, for modulating measured patterns.

In SIM, a sinusoidal modulated intensity pattern is used by moving spectrum for capturing the high frequency data. A twofold resolution imaging can be achieved by SIM $[11,12]$. Here the incident laser beam is parallel to the

\footnotetext{
${ }^{1}$ Center of Ultra-precision Optoelectronic Instrument Engineering, Harbin Institute of Technology, Harbin 150080, China

${ }^{2}$ Key Lab of Ultra-precision Intelligent Instrumentation (Harbin Institute of Technology), Ministry of Industry and Information Technology, Harbin 150080, China

${ }^{3}$ Department of Electrical Engineering, California Institute of Technology, Pasadena, CA 91125, USA

${ }^{4}$ Department of Physics, Harbin Institute of Technology, Harbin 150001, China

*E-mail: zjliu@hit.edu.cn
} 
optical axis [13]. The collimated light restricts the range of spatial spectrum $[14,15]$. Nonlinear SIM $[16,17,18]$ was invented to promote resolution. Coincidentally, the saturated structured illumination microscopy (SSIM) $[19,20]$ was introduced to improve resolution by utilizing the nonlinear effect of fluorescent molecules. SSIM, however, is limited by fluorescent labelling. Some samples in SSIM are easily damaged by high intensity laser $[21,22,23,24]$.

In this work, tilt illumination is introduced to SIM (tiSIM) for enhancing resolution. The mathematical model of tilt illumination is an exponential function in object reconstruction. This function is used to shift spectrum. The immesurable frequency compononts are moved into the passband of OTF. The proposed method can make the resolution enhancement as a nonlinear SIM. The theoretical analysis and simulation results are given to validate the performance of tiSIM.

\section{Method}

In SIM, the reconstruction of high-resolution image requires multiple measured patterns at different phase shifts along carrier frequency directions. The grating centers on the optical axis and rotates specific angles, such as $0^{\circ}, 45^{\circ}, 90^{\circ}$, and $135^{\circ}$. At each rotation angle of the grating, 0 , and \pm 1 orders of diffraction light are used to illuminate the sample simultaneously. Here \pm 1 order diffraction beam illuminating sample is equivalent to moving the sample spectrum in two opposite directions. To realize super-resolution imaging, it is necessary to decouple the spectra from $0, \pm 1$ orders of diffraction patterns $[21,22,23,24]$.

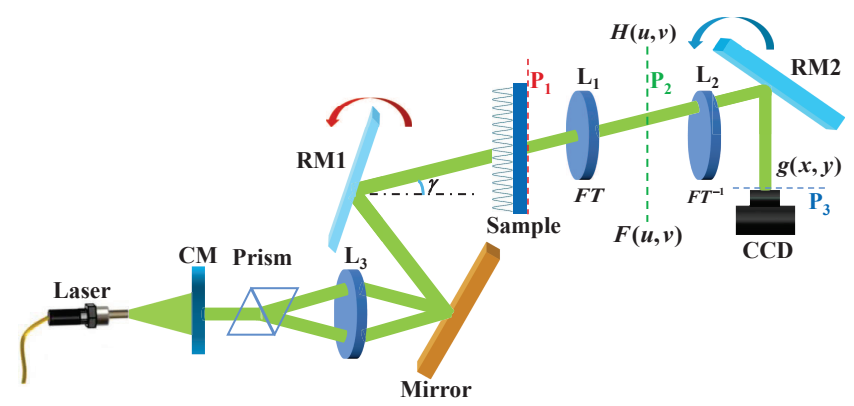

Fig. 1 A schematic of experimental system for tiSIM. CM: collimating mirror; $\mathrm{L}_{1}, \mathrm{~L}_{2}$, $\mathrm{L}_{3}$ : lens; RM: rotatable mirror. RM1 and RM2 achieve the tilt illumination. $\gamma$ is the tilt angle between the incident light and the optical axis marked with chain line. $F T$ and $F T^{-1}$ represent Fourier transform and its inverse.

In Fig. 1, a schematic diagram of tiSIM is illustrated. Two lens $\left(\mathrm{L}_{1}\right.$ and $\left.\mathrm{L}_{2}\right)$ constitute a 4 f system. $\mathrm{P}_{1}, \mathrm{P}_{2}$ and $\mathrm{P}_{3}$ are object plane, frequency plane and imaging plane, respectively. The incident light is divided into two parts by the prism. Two beams interfere to form the sinusoidal stripe as the illumination pattern playing the role of the grating. The beam reflected by RM1 has a tilt 
angle with the optical axis and projected onto the grating with modulated transmittance. The tilt angle $\gamma$ can be adjusted by rotating RM1. RM2 is applied to ensure that the output beam can propagate vertically to CCD.

Several pictures are generated by moving a grating for reconstructing a high resolution image $[25,26,27,28]$. The grating function is expressed as follows

$$
m(x, y)=\cos \left[2 \pi k_{0}(x \cos \theta+y \sin \theta)+\alpha\right],
$$

where $\theta$ is the rotation angle of sinusoidal stripe. $\alpha$ represents a phase shift. The carrier frequency $k_{0}$ is equal to the cutoff frequency $f_{c}$. The imaging model of SIM is expressed as

$$
g(x, y)=[s(x, y) \cdot m(x, y)] \otimes h(x, y),
$$

where $h(x, y)$ represents the point spread function (PSF) as follows

$$
h(x, y)=\operatorname{circ}\left(\frac{\sqrt{x^{2}+y^{2}}}{f_{c}}\right) .
$$

The imaging process of tiSIM is written as

$$
g(x, y)=[s(x, y) \cdot \exp (\mathrm{i} k x \cos \gamma) \cdot m(x, y)] \otimes h(x, y) .
$$

where the exponential term $\exp (\mathrm{i} k x \cos \gamma)$ is from tilt illumination. tiSIM in frequency domain is written as

$$
\left[\begin{array}{l}
I_{1} \\
I_{2} \\
I_{3}
\end{array}\right]=\frac{1}{2} M_{\phi} M_{H} M_{S},
$$

where

$$
\begin{gathered}
M_{\phi}=\left[\begin{array}{lll}
2 & e^{-\mathrm{i} \phi_{1}} & e^{\mathrm{i} \phi_{1}} \\
2 & e^{-\mathrm{i} \phi_{2}} & e^{\mathrm{i} \phi_{2}} \\
2 & e^{-\mathrm{i} \phi_{3}} & e^{\mathrm{i} \phi_{3}}
\end{array}\right], \\
M_{H}=\left[\begin{array}{ccc}
H & 0 & 0 \\
0 & H & 0 \\
0 & 0 & H
\end{array}\right],
\end{gathered}
$$

and

$$
M_{S}=\left[\begin{array}{c}
S(\mathbf{k}-\cos \gamma / \lambda) \\
S\left(\mathbf{k}+\mathbf{k}_{0}-\cos \gamma / \lambda\right) \\
S\left(\mathbf{k}-\mathbf{k}_{0}-\cos \gamma / \lambda\right)
\end{array}\right],
$$

where $I_{n}$ represents the spectrum of image received by CCD. The symbol $S$ indicates the spectrum corresponding to 0 , and \pm 1 orders of diffraction light. $H$ is OTF. The parameters $\mathbf{k}$ and $\phi_{n}$ denote the spatial frequency and the phase shift. Selecting different values of $\phi_{n}$, a system of linear matrix equations can be obtained to solve the sample spectrum.

The images in Fig. 2 are obtained by wide field (WF) imaging and SIM. The resolution of wide field imaging can be calculated as $\sigma_{\mathrm{WF}}=0.61 \lambda / \mathrm{NA}=$ 

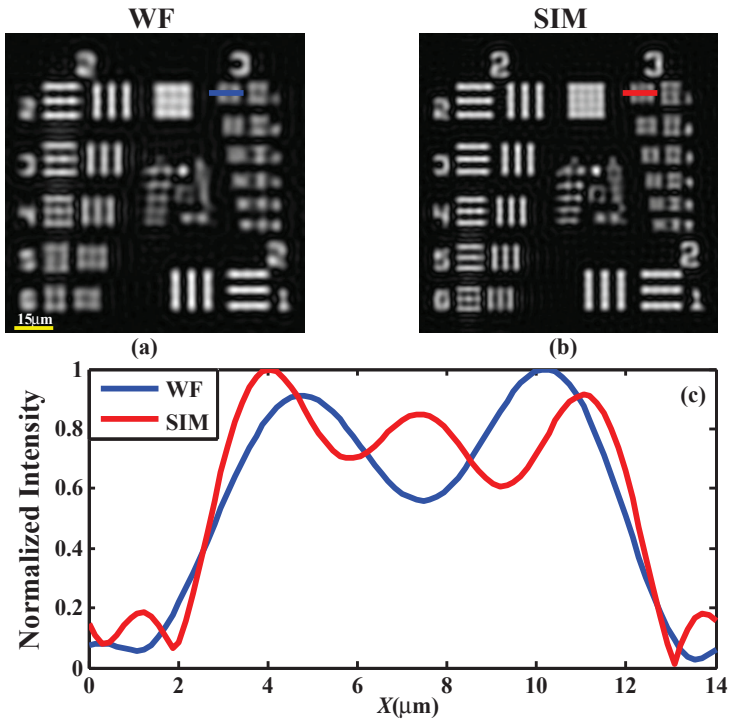

Fig. 2 The imaging results: (a) WF, (b) SIM, (c) normalized intensity profile in the area specified by blue and red lines. The yellow scale bar in (a) indicates $15 \mu \mathrm{m}$.

$3.86 \mu \mathrm{m}$. Here the wavelength of incident light is $632.8 \mathrm{~nm}$. Numerical aperture (NA) is 0.1 . The resolution of SIM is $\sigma_{\mathrm{SIM}}=1.93 \mu \mathrm{m}$, since strutured illumination has 2 -fold resolution. The distance between the vertical lines specified by blue and red lines in Fig. 2(a) and 2(b) is $2 \mu \mathrm{m}$. Here the three vertical lines can only be resolved by SIM rather than wide field imaging because $2 \mu \mathrm{m}$ is between $\sigma_{\mathrm{WF}}$ and $\sigma_{\mathrm{SIM}}$. The resolution of WF and SIM can be validated by blue and red lines in Fig. 2(c).
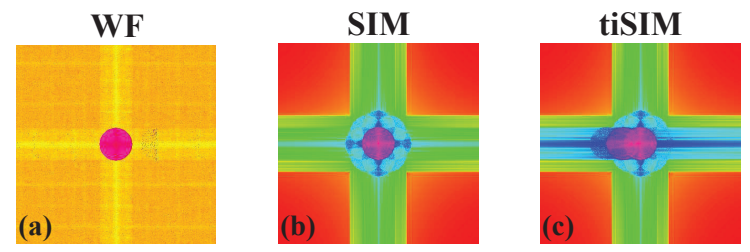

Fig. 3 The spectrum maps: (a) WF, (b) SIM, (c) tiSIM with $\gamma=10^{\circ}$. The area of frequency spectrum becomes larger from left to right.

In Fig. 3, three images are the sample frequency spectrums of WF, SIM and tiSIM. The spectrum from tiSIM in Fig. 3(c) is larger than the one in Fig. 3(b). The more infromation of spectrum can be carried to OTF of tiSIM, which is beneficial for high-resolution reconstruction. The operations of tiSIM are divided to two parts. Firstly, the incident light is parallel to the optical axis. Secondly, tilt illumination is performed to rich the spectrum. 


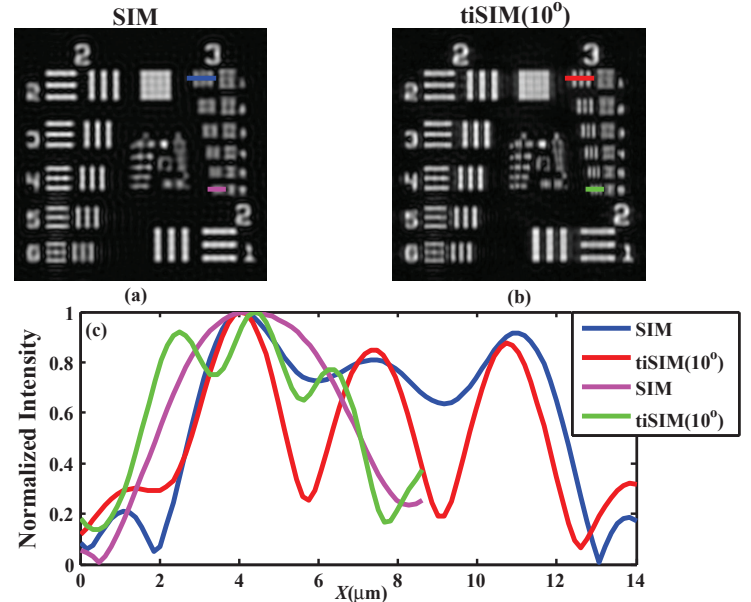

Fig. 4 The recovery images: (a) SIM, (b) tiSIM $\left(10^{\circ}\right)$, (c) normalized intensity from the area specified by the same colour lines. The resolutions of SIM and tiSIM are $1.93 \mu \mathrm{m}$ and $0.9 \mu \mathrm{m}$.

In Fig. 4(a) and 4(b), the images recovered by SIM and tiSIM (10 $)$, are displayed. The blue and green structures specified by the same color lines in reconstructed images are the diffraction limits of SIM and tiSIM $\left(10^{\circ}\right)$. From Fig. 4, tiSIM can enhance the resolution of reconstructed image.

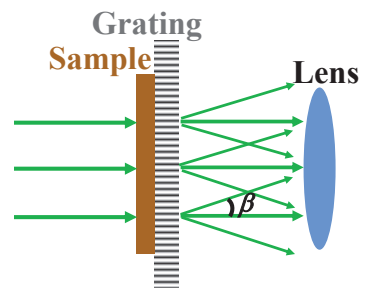

(a)

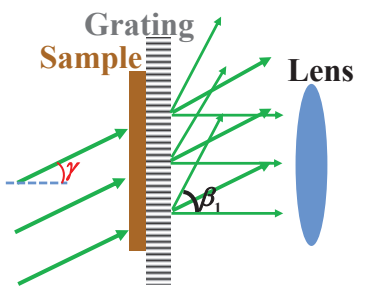

(b)

Fig. 5 The inclination angle and aperture angle: (a) in SIM, (b) in tiSIM. The inclination angle is limited by the aperture of $4 \mathrm{f}$ system.

In SIM, the numerical aperture, $\mathrm{NA}_{\mathrm{SIM}}$ is defined as

$$
\mathrm{NA}_{\mathrm{SIM}}=n \cdot \sin \beta=\lambda \cdot k_{0},
$$

where $n$ is the refractive index of the medium. $\beta$ is the half of the aperture angle in Fig. 5(a). The grating equation is $(\sin \beta) / k_{0}=k \lambda,(k=0, \pm 1, \pm 2$, ...). The period is defined as $d=1 / k_{0}$ and $k$ is the diffraction order. In tiSIM, $\mathrm{NA}_{\text {tisim }}$ is defined as

$$
\mathrm{NA}_{\mathrm{tiSIM}}=n \cdot \sin \beta_{1}=\lambda \cdot k_{0}+\sin \gamma
$$


Thereby, the resolution ratio between SIM and tiSIM is expressed as

$$
\eta=\frac{\sigma_{\mathrm{SIM}}}{\sigma_{\mathrm{tiSIM}}}=\frac{\mathrm{NA}_{\mathrm{tiSIM}}}{\mathrm{NA}_{\mathrm{SIM}}}=1+\frac{\sin \gamma}{\lambda \cdot k_{0}},
$$

where $k_{0}=0.1 /(0.61 \lambda)$.

Considering the aperture of $4 \mathrm{f}$ system, the angle $\gamma$ is limited as follows

$$
\begin{gathered}
-\frac{D}{4 f \lambda} \leq-k_{0}+\frac{\cos \gamma}{\lambda} \leq \frac{D}{4 f \lambda}, \\
\gamma_{\max }=\arccos \left(\frac{D}{4 f}\right),
\end{gathered}
$$

where $D$ is the minimum aperture radius of $4 \mathrm{f}$ system.
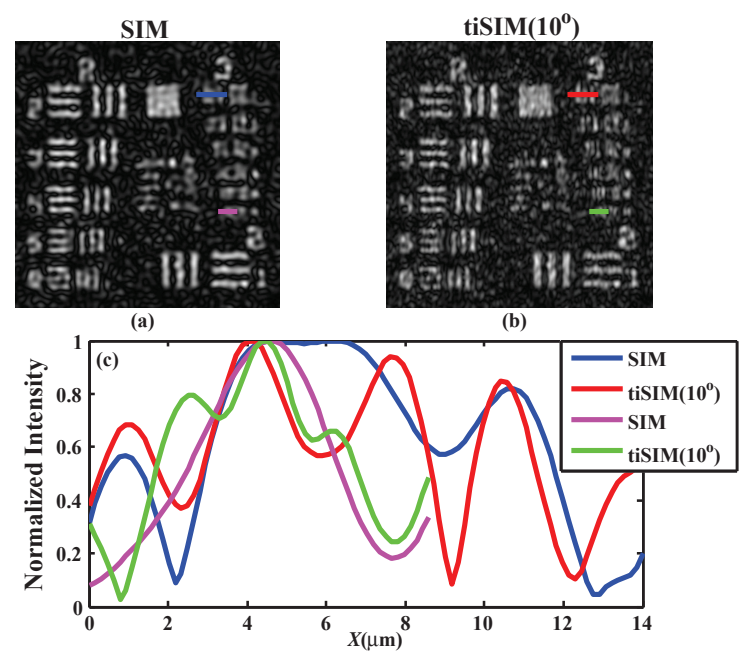

Fig. 6 The noise robustness with Gaussian white noise: (a) by SIM, (b) by tiSIM $\left(10^{\circ}\right)$, (c) the blue, purple, green and red curves are normalized intensity of the area specified by the same color lines in (a) and (b).

Noise robustness of image reconstruction is tested by tiSIM and SIM. Here Gaussian white noise is added for simulating experimental case. In Fig. 6, two retrieved images by SIM and tiSIM $\left(10^{\circ}\right)$ are shown. Despite the influence of Gaussian white noise, the three vertical lines specified by the green lines can still be differentiated in Fig. 6(b). From Fig. 6, tiSIM has higher noise robustness than SIM.

\section{Multi-angle tilt illumination}

To improve the imaging resolution of tiSIM, multi-angle tilt illumination is employed. Here NA of the optical system is enhanced. In Fig. 7, the spectrum 
of the recovered images by $\operatorname{tiSIM}\left(10^{\circ}\right)$ and $\operatorname{tiSIM}\left(10^{\circ}, 15^{\circ}, 20^{\circ}, 30^{\circ}\right)$ is illustrated. In Fig. 7(b), a bigger area of spectrum is obtained and can be used for high-resolution reconstruction.

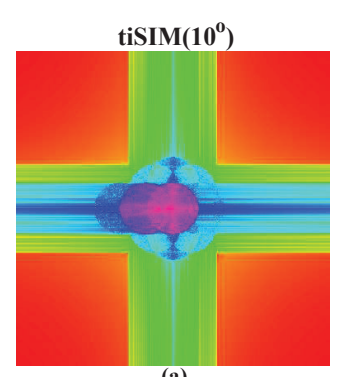

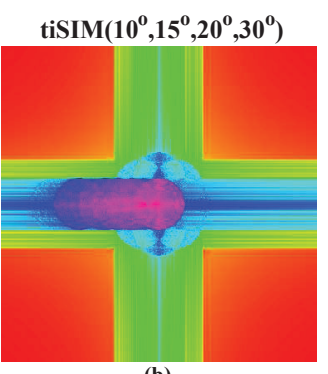

Fig. 7 The frequency spectrums of the recovery images by tiSIM $\left(10^{\circ}\right)$ and tiSIM $\left(10^{\circ}\right.$, $\left.15^{\circ}, 20^{\circ}, 30^{\circ}\right)$. Here the parts in dark circular closure curves are frequency spectrum.

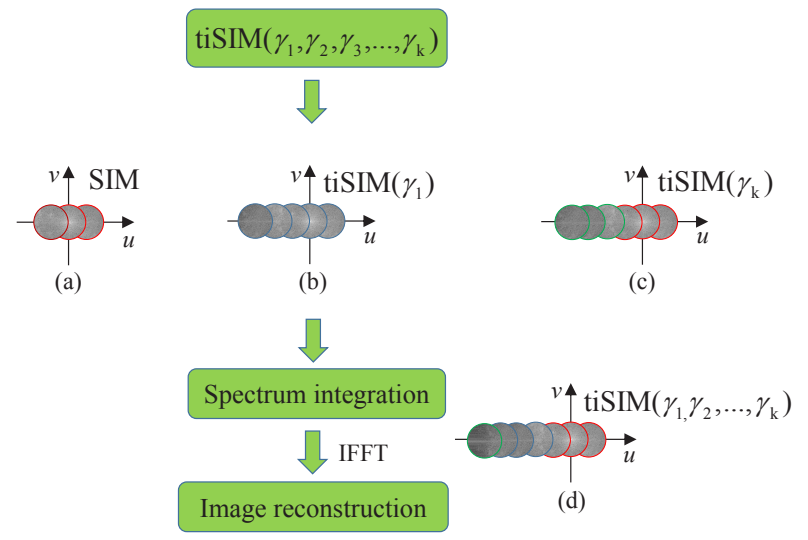

Fig. 8 A flowchart of tiSIM with multi-angle illumination. (a)-(c) represent the spectrum generated by $\operatorname{SIM}, \operatorname{tiSIM}\left(\gamma_{1}\right)$ and $\operatorname{tiSIM}\left(\gamma_{k}\right)$. (d) is an integrated spectrum.

The flowchart of tiSIM with multi-angle illumination in is displayed in Fig. 8. At each angle, the spectrum is calculated by Eq. (5). In Fig. 8(a-c), the spectra obtained by SIM, tiSIM $\left(\gamma_{1}\right)$, and tiSIM $\left(\gamma_{k}\right)$ are given. In Fig. $8(d)$ the integrated spectrum with several angles is shown. From Fig. 8, more data in high frequency range of spectrum is moved into the pass band of OTF by tiSIM with more angles. In Fig. 9, the images are calculated by tiSIM with multi-angle illumination. From Fig. 9(c), the resolutions are $0.9 \mu \mathrm{m}$ and 0.5 $\mu \mathrm{m}$ for the two cases. It shows that multi-angle tilt illumination can improve resolution by collecting spectrum from more directions. 

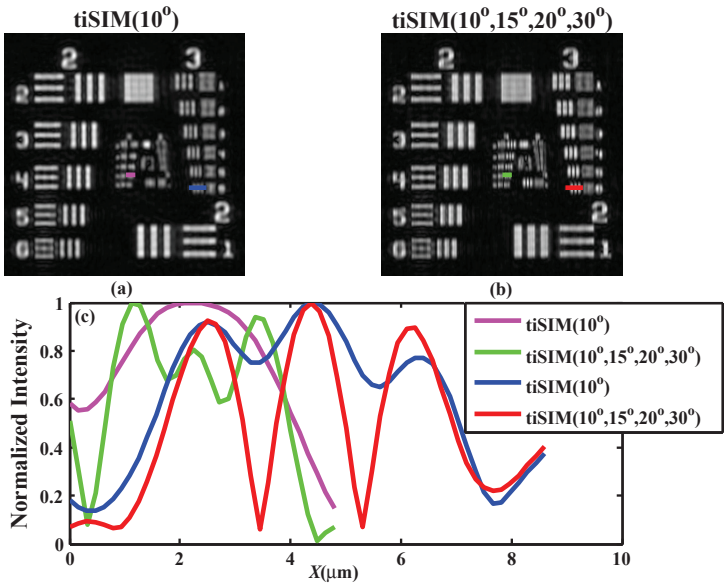

Fig. 9 The reconstructed images: (a) by tiSIM $\left(10^{\circ}\right)$, (b) $\operatorname{tiSIM}\left(10^{\circ}, 15^{\circ}, 20^{\circ}, 30^{\circ}\right)$, (c) normalized intensity profiles from specified range.

In tiSIM, inclination angle error is considered for experiment. In Fig. 10(a, b) are the images made by tiSIM under the condition that all inclination angle errors of tilt illumination are $2^{\circ}$. The three vertical lines specified by the blue lines are not able to be differentiated in Fig. 10(a) under the influence of the errors by compared with the same area in Fig. 4(a). The different contrast between blue and green curves in Fig. 4(c) and Fig. 10(c) also proves the different angular errors robustness.

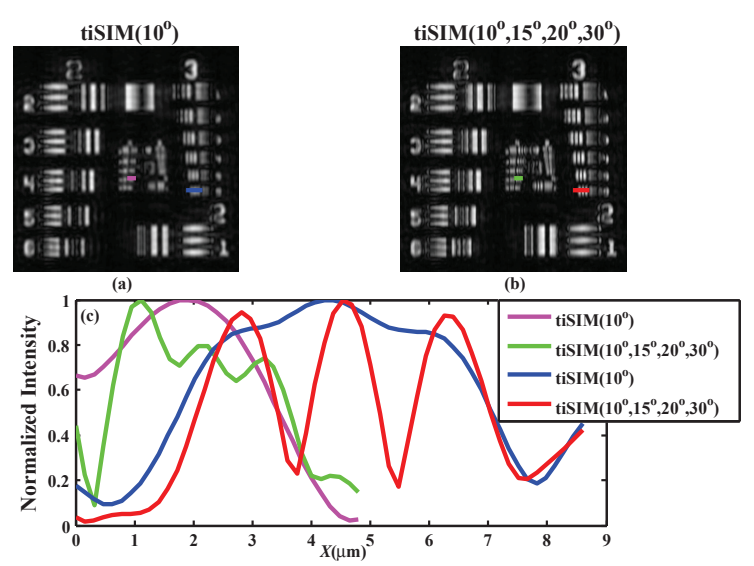

Fig. 10 The recovery images by tiSIM under the condition that the inclination angle error: (a) single angle, (b) four angles, (c) normalized intensity curves from the area specified by the same color lines. 


\section{Comparison with nonlinear SIM}

Nonlinear SIM (NSIM) is put forward by applying photoswitchable protein instead of fluorescence saturation for super-resolution imaging [29,30,31,32]. The sample frequency spectrum and the recovery image in Fig. 11 are obtained by WF, SIM, and third-order NSIM. The third-order NSIM can obtain much more high frequency spectrum. tiSIM is by rotating illumination beam to detect high frequency without high density laser.

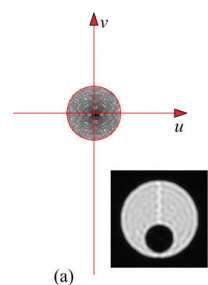

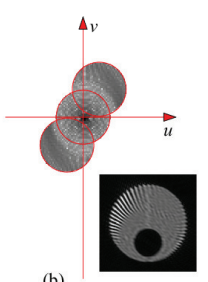

(b)

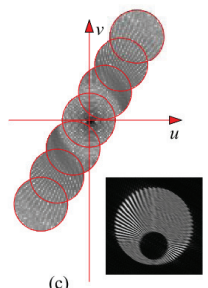

Fig. 11 Spectra and reconstructed images: (a) by WF, (b) by SIM, (c) by third-order NSIM.

\section{Conclusion}

In SIM, the parallel incident light restricts the high frequency information carried to OTF. Tilt illumination is employed to SIM for expanding the spectrum detection range. More data of high frequency can be integrated for superresolution imaging. The proposed method has higher noise robustness. In addition, SIM with multi-angle illumination has higher angular error robustness.

\section{Acknowledgments}

This work was supported by the National Natural Science Foundation of China (Nos. 11874132, 61975044, and 12074094).

\section{References}

1. Gustafsson, M.G.: Surpassing the lateral resolution limit by a factor of two using structured illumination microscopy. J. Microsc. 198, 82-87 (2000)

2. Shao, L., Winoto, L., Agard, D.A., Gustafsson, M.G., Sedat, J.W.: Interferometer-based structured-illumination microscopy utilizing complementary phase relationship through constructive and destructive image detection by two cameras. J. Microsc. 246, 229-236 (2012)

3. Gustafsson, M.G., Shao, L., Carlton, P.M., Wang, C.J., Golubovskaya, I.N., Cande, W.Z., Agard, D.A., Sedat, J.W.: Three-dimensional resolution doubling in wide-field fluorescence microscopy by structured illumination. Biophys. J. 94, 4957-4970 (2008) 
4. Shao, L., Kner, P., Rego, E., Gustafsson, M.G.: Super-resolution 3D microscopy of live whole cells using structured illumination. Nat. Methods 8, 1044-1046 (2011).

5. Chen, J., Xu, Y., Lv, X., Lai, X., Zeng, S.: Super-resolution differential interference contrast microscopy by structured illumination. Opt. Express 21, 112-121 (2013)

6. Grebenyuk, A.A., Ryabukho, V. P.: Numerically focused optical coherence microscopy with structured illumination aperture. Comput. Opt. 42, 248-253 (2018)

7. Saxena, M., Eluru, G., Gorthi, S.S.: Structured illumination microscopy. Adv. Opt. Photon. 7, 241-275 (2015)

8. Lu, R.W., Wang, B.Q., Zhang, Q.X., Yao, X.C.: Super-resolution scanning laser microscopy through virtually structured detection. Biomed. Opt. Express 4, 1673-1682 (2013)

9. Jin, X., Ding, X., Tan, J., Yao, X., Shen, C., Zhou, X., Tan, C., Liu, S., Liu, Z.: Structured illumination imaging without grating rotation based on mirror operation on 1D Fourier spectrum. Opt. Express 273, 2016-2028 (2019)

10. Ni, H., Zou, L., Guo, Q., Ding, X.: Lateral resolution enhancement of confocal microscopy based on structured detection method with spatial light modulator. Opt. Express 25, 2872-2882 (2017)

11. Lee, W.S., Lim, G., Kim, W.C., Choi, G.J., Yi, H.W., Park, N.C.: Investigation on improvement of lateral resolution of continuous wave STED microscopy by standing wave illumination. Opt. Express 26, 9901-9919 (2018)

12. Huttunen, M.J., Abbas, A., Upham, J., Boyd, R.W.: Label-free super-resolution with coherent nonlinear structured-illumination microscopy. J. Opt. 19, 085504 (2017).

13. Chowdhury, S., Izatt, J.: Structured illumination quantitative phase microscopy for enhanced resolution amplitude and phase imaging. Biomed. Opt. Express 4, 1795-1805 (2013)

14. Dong, S., Nanda, P., Guo, K., Liao, J., Zheng, G.: Incoherent Fourier ptychographic photography using structured light. Photon. Res. 3, 19-23 (2005)

15. Jost, A., Tolstik, E., Feldmann, P., Wicker, K., Sentenac, A., Heintzmann, R.: Optical sectioning and high resolution in single-slice structured illumination microscopy by thick slice blind-SIM reconstruction. PLoS One 10, e0132174 (2015)

16. Refo, E., Shao, L., Macklin, J., Winoto, L., Johansson, G., Hughes, N., Davidson, M., Gustafsson, M.G.: Nonlinear structured-illumination microscopy with a photoswitchable protein reveals cellular structures at 50-nm resolution. Proc. Natl. Acad. Sci. 109, E135E143 (2012)

17. Mou, Z., Lu, X., Lv, H., Han, Y., Yue, Q., Wang, S., Teng, S.:Metasurface array illuminator based on Fresnel holography. Opt. Lasers Eng. 131, 106146 (2020)

18. Cheng, L., Lien, C., Sie, Y., Hu, Y., Lin, C., Chien, F., Xu, C., Dong, C., Chen, S. Nonlinear structured-illumination enhanced temporal focusing multiphoton excitation microscopy with a digital micromirror device. Biomed. Opt. Express 5, 2526-2536 (2014)

19. Gustafsson, M.G.: Nonlinear structured-illumination microscopy: wide-field fluorescence imaging with theoretically unlimited resolution. Proc. Natl. Acad. Sci. 102, 13081-13086 (2005)

20. Cao, R., Kuang, C., Liu, Y., Liu, X.: 2017 Superresolution via saturated virtual modulation microscopy. Opt. Express 25, 32364-32379 (2017)

21. Gao, L., Bedard, N., Hagen, N., Kester, R., Tkaczyk, T.: Depth-resolved image mapping spectrometer (IMS) with structured illumination. Opt. Express 19, 17439-17452 (2011)

22. Kner, P., Chhun, B., Griffis, E., Winoto, L., Gustafsson, M.G.: 2009 Super-resolution video microscopy of live cells by structured illumination. Nat. Methods 6, 339-342 (2009)

23. Wicker, K., Mandula, O., Best, G., Fiolka, R., Heintzmann, R.: Phase optimization for structured illumination microscopy. Opt. Express 21, 2032-2049 (2013)

24. Jones, S.A., Shim, S.H., He, J., Zhuang, X.: Fast, three-dimensional super-resolution imaging of live cells. Nat. Methods 8, 499-505 (2011)

25. Cai, Z., Liu, X., Pedrini, G., Osten, W., Peng, X.: Structured-light-field 3D imaging without phase unwrapping. Opt. Lasers Eng. 129, 106047 (2020)

26. Hong, W., Wang, J., Li, W.: CSK hopping pattern model for visible light communication networks. Opt. Quant. Electron. 41, 108 (2019)

27. Righolt, C.H., Slotman, J.A., Young, I.T., Mai, S., Vliet, L.J., Stallinga, S.: Image filtering in structured illumination microscopy using the lukosz bound. Opt. Express 21, 24431-24451 (2013) 
28. Gao, Z., Cheng, X., Zhang, L., Hu, Y., Hao, Q.: Compressive ghost imaging in scattering media guided by region of interest. J. Opt. 22, 055704 (2020)

29. Heintzmann, R., Jovin, T.M., Cremer, C.: Saturated patterned excitation microscopy-a concept for optical resolution improvement. J. Opt. Soc. Am. A 19, 1599-1609 (2002)

30. Hassanien, A.M., Atta, A.A., El-Nahass, M.M., Ahmed, S.I., Shaltout, A.A., Al-Baradi, A.M., Alodhayb, A., Kamal, A.M.: Effect of annealing temperature on structural and optical properties of gallium oxide thin films deposited by RF-sputtering. Opt. Quant. Electron. 52, 194 (2020).

31. Lin, Q., Wang, D., Wang, Y., Rong, L., Zhao, J., Guo, S., Wang, M.: Super-resolution imaging by microsphere-assisted optical microscopy. Opt. Quant. Electron. 48, 557 (2016)

32. Hu, Z., Tan, C., Song, Z., Liu, Z.: A coherent diffraction imaging by using an iterative phase retrieval with multiple patterns at several directions. Opt. Quant. Electron. 52, 29 (2020) 


\section{Figures}

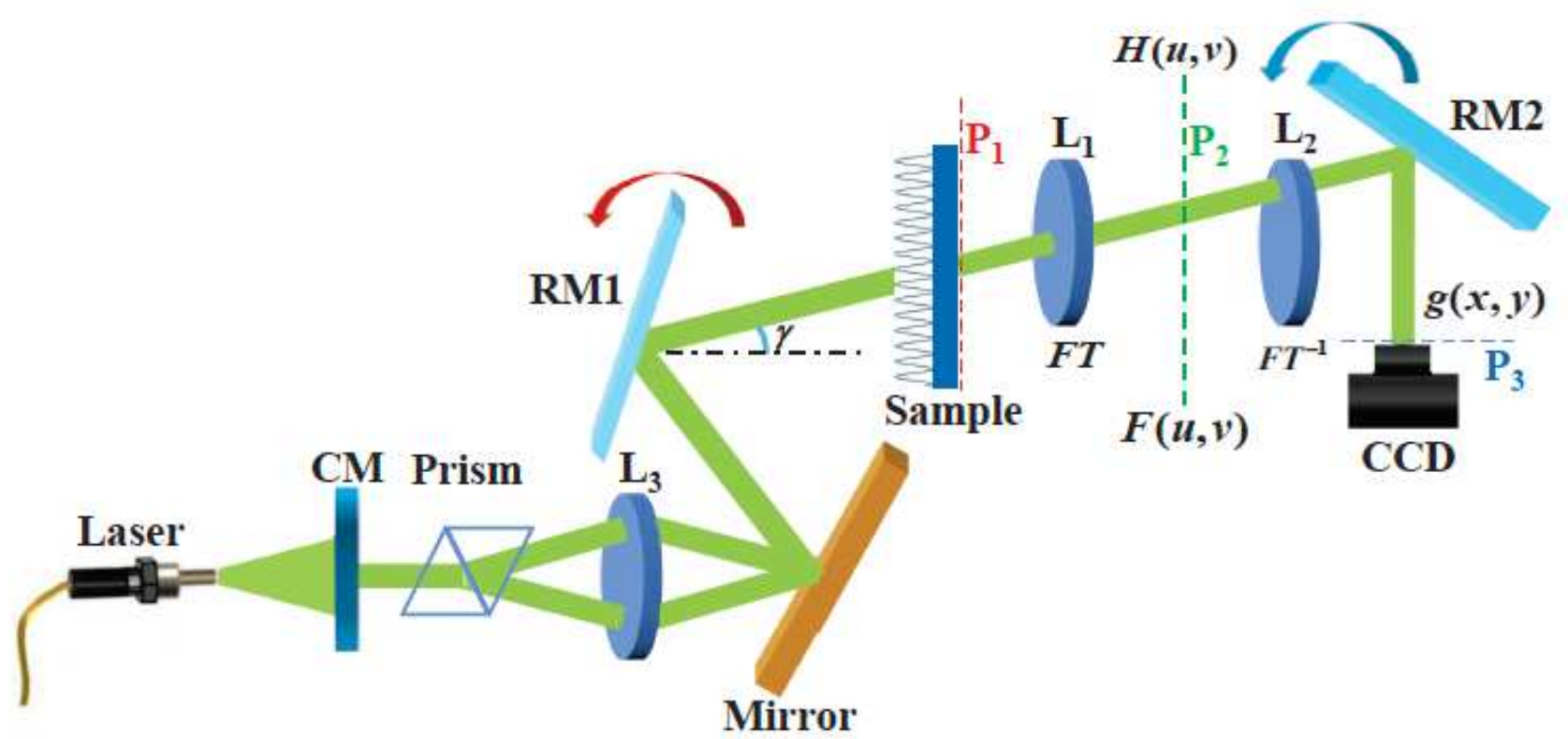

\section{Figure 1}

A schematic of experimental system for tiSIM. CM: collimating mirror; L1, L2, L3: lens; RM: rotatable mirror. RM1 and RM2 achieve the tilt illumination. $\gamma$ is the tilt angle between the incident light and the optical axis marked with chain line. FT and FT-1 represent Fourier transform and its inverse. 


\section{WF}

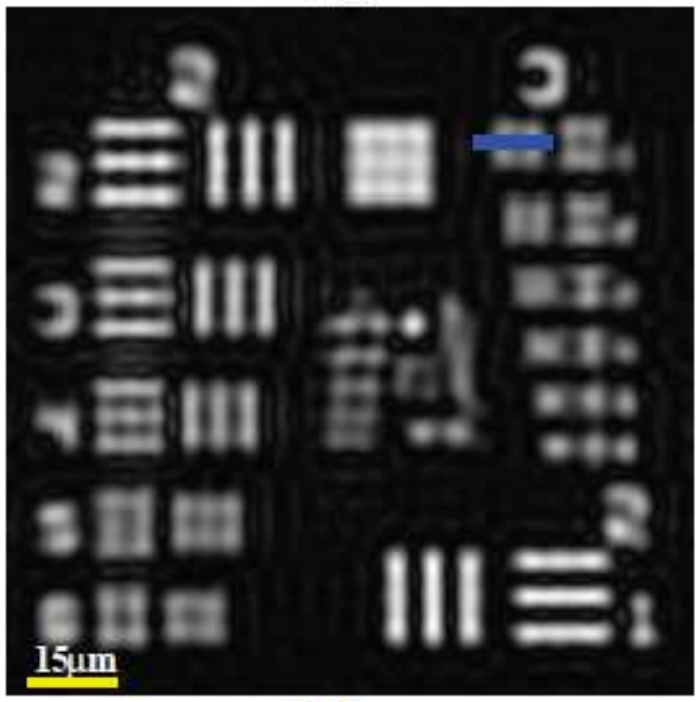

(a)

\section{SIM}

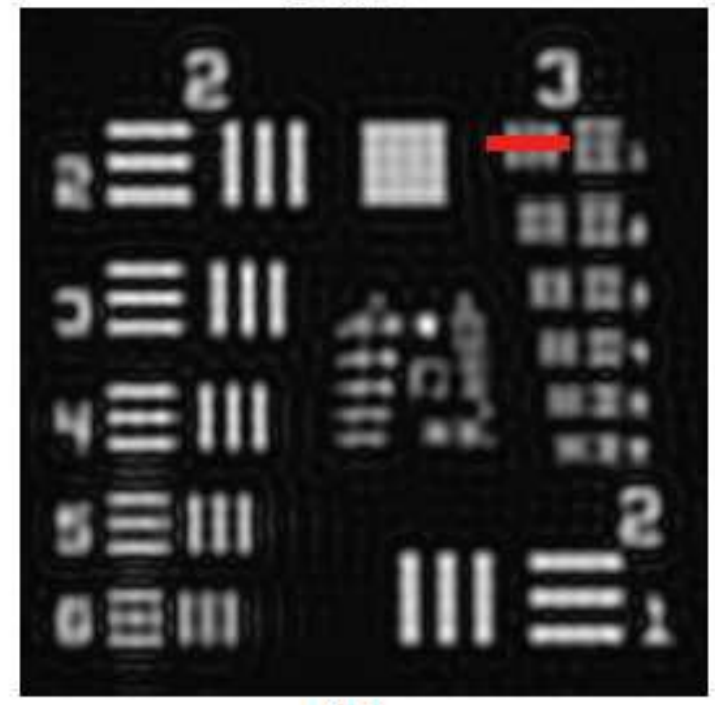

(b)

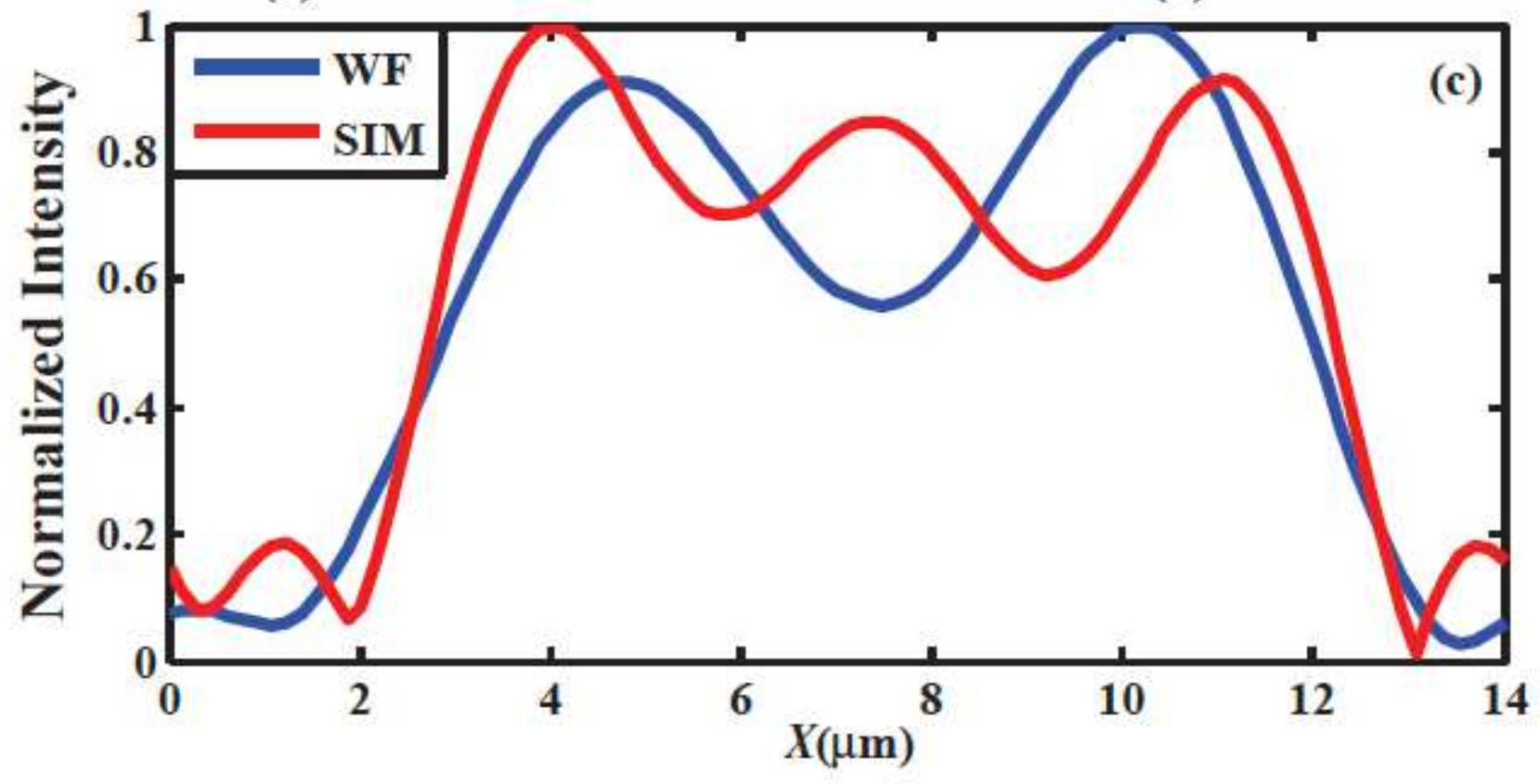

Figure 2

The imaging results: (a) WF, (b) SIM, (c) normalized intensity profile in the area specified by blue and red lines. The yellow scale bar in (a) indicates $15 \mu \mathrm{m}$. 

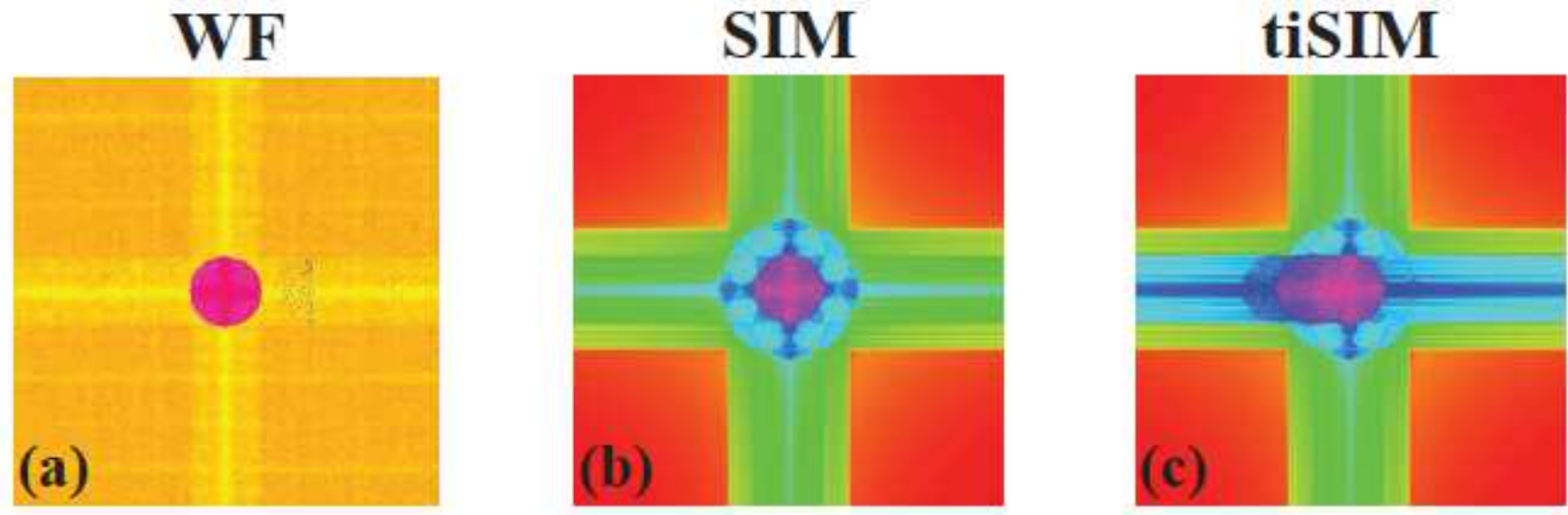

Figure 3

The spectrum maps: (a) WF, (b) SIM, (c) tiSIM with $y=10^{\circ}$. The area of frequency spectrum becomes larger from left to right.

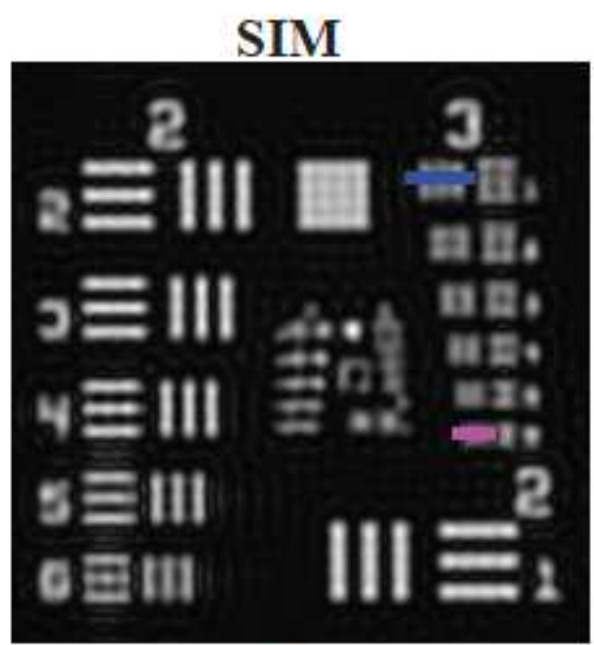

(a)

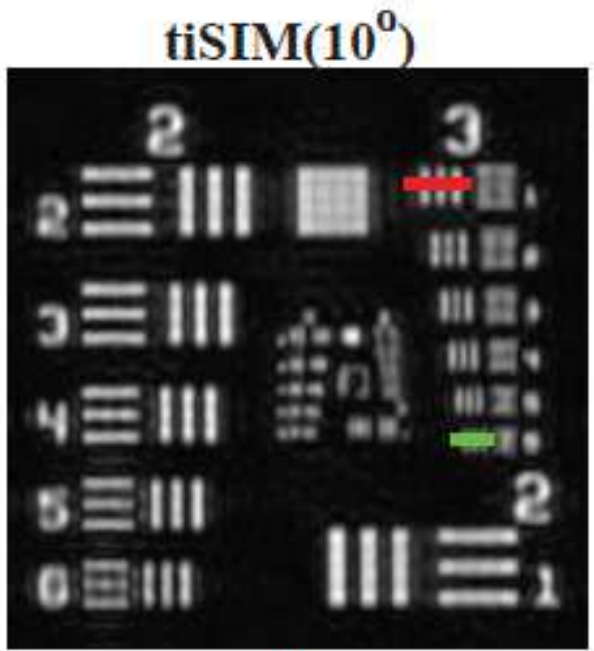

(b)

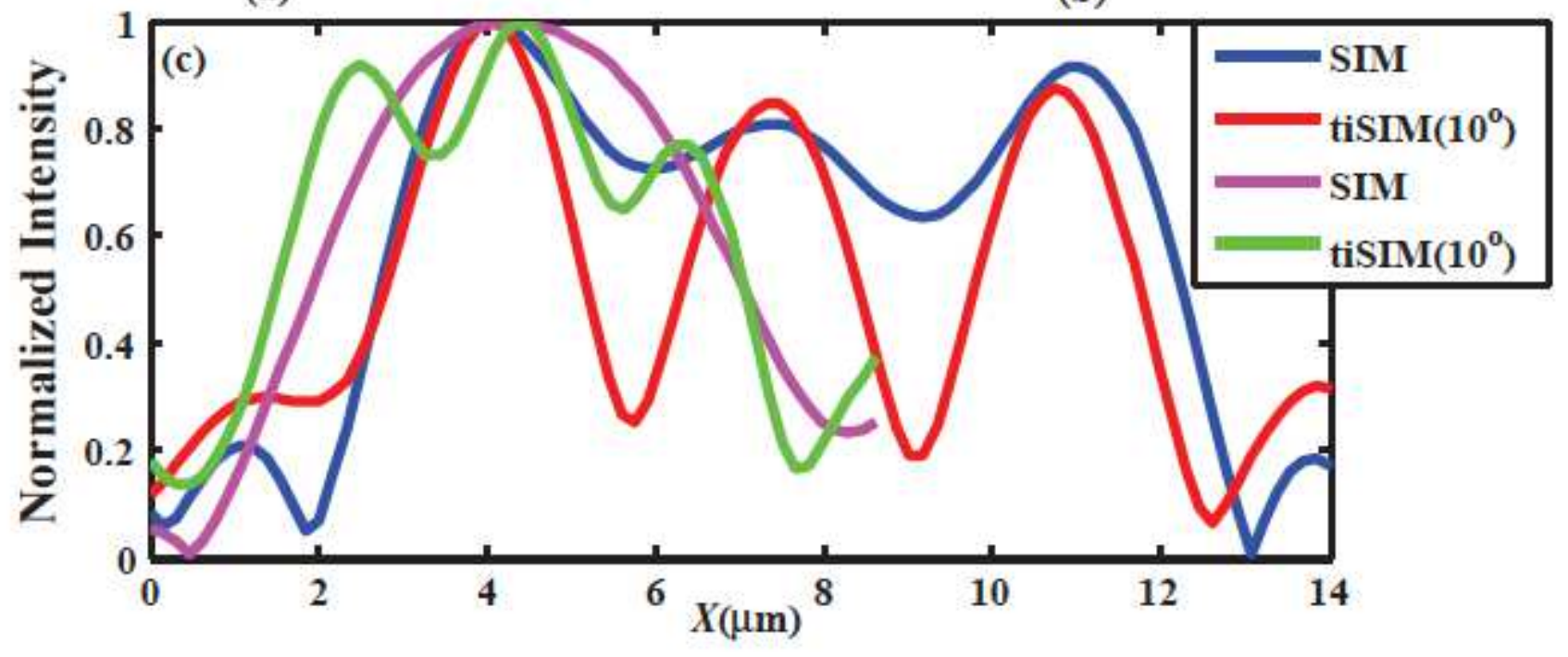


Figure 4

The recovery images: (a) SIM, (b) tiSIM $\left(10^{\circ}\right)$, (c) normalized intensity from the area specified by the same colour lines. The resolutions of SIM and tiSIM are $1.93 \mu \mathrm{m}$ and $0.9 \mu \mathrm{m}$.

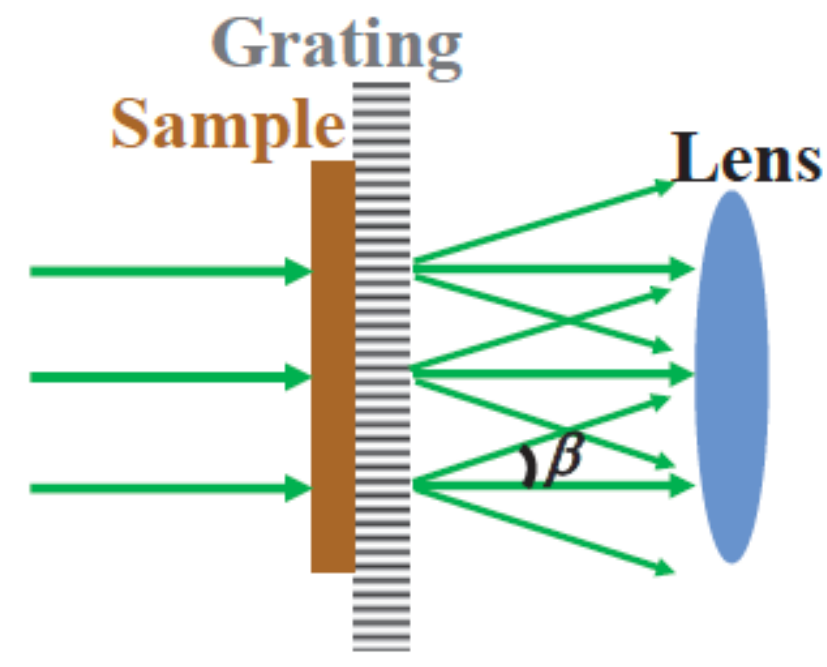

(a)

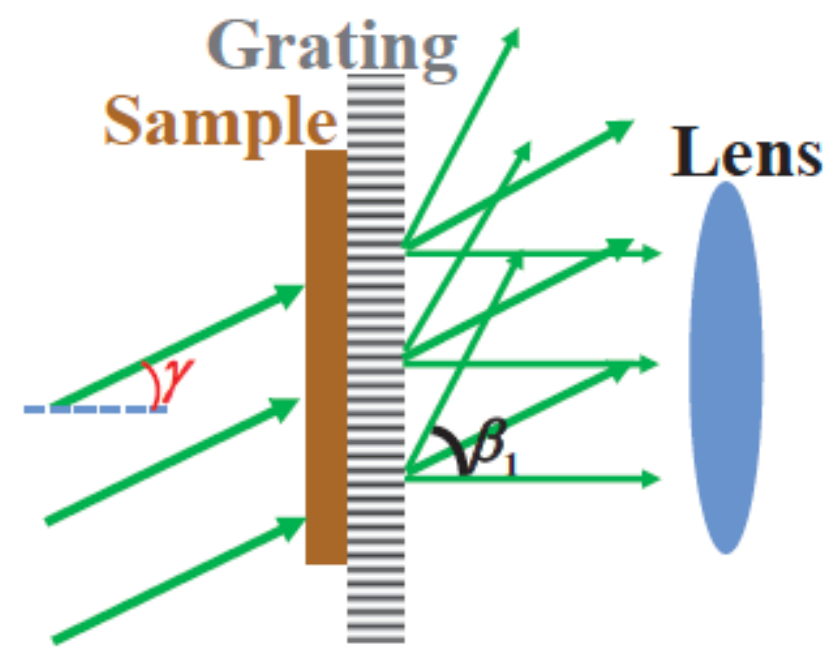

(b)

\section{Figure 5}

The inclination angle and aperture angle: (a) in SIM, (b) in tiSIM. The inclination angle is limited by the aperture of $4 \mathrm{f}$ system. 


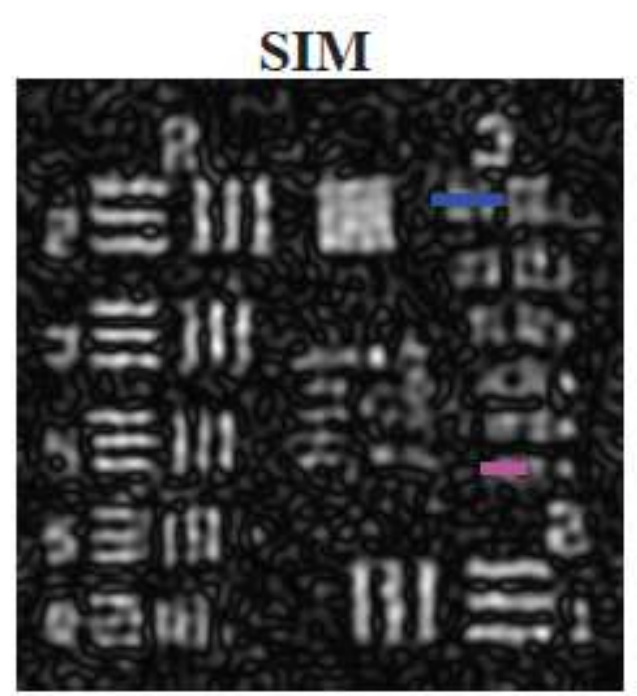

(a)

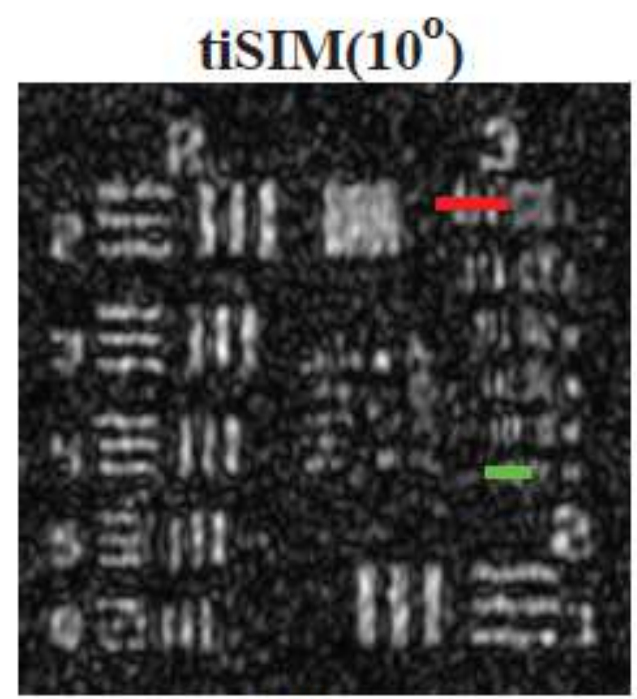

(b)

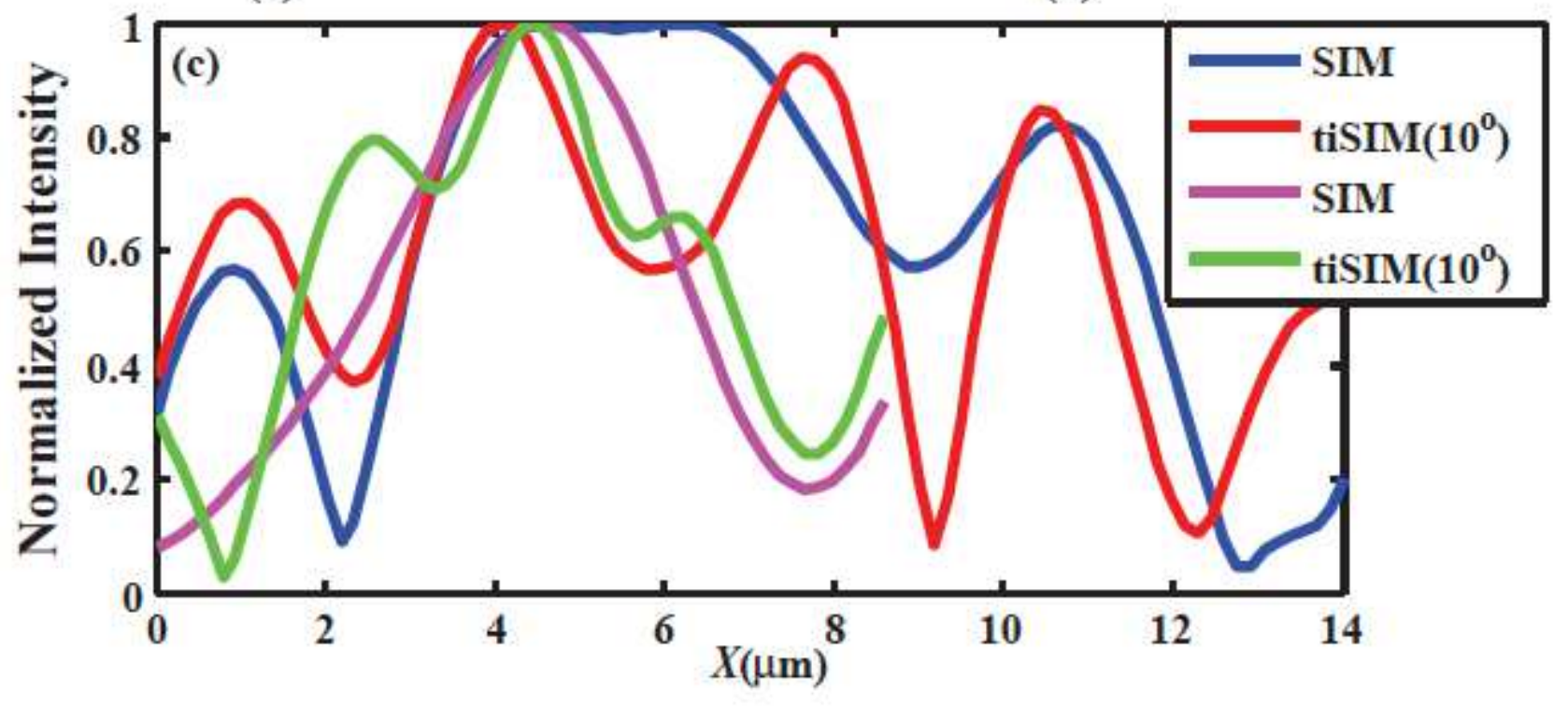

Figure 6

The noise robustness with Gaussian white noise: (a) by SIM, (b) by tiSIM $\left(10^{\circ}\right)$, (c) the blue, purple, green and red curves are normalized intensity of the area specified by the same color lines in (a) and (b). 


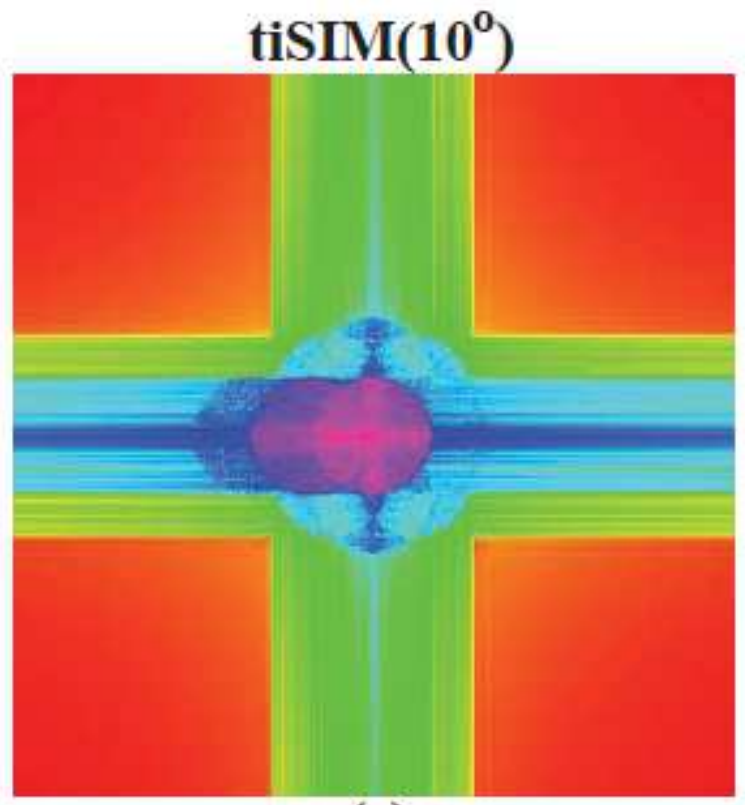

(a)

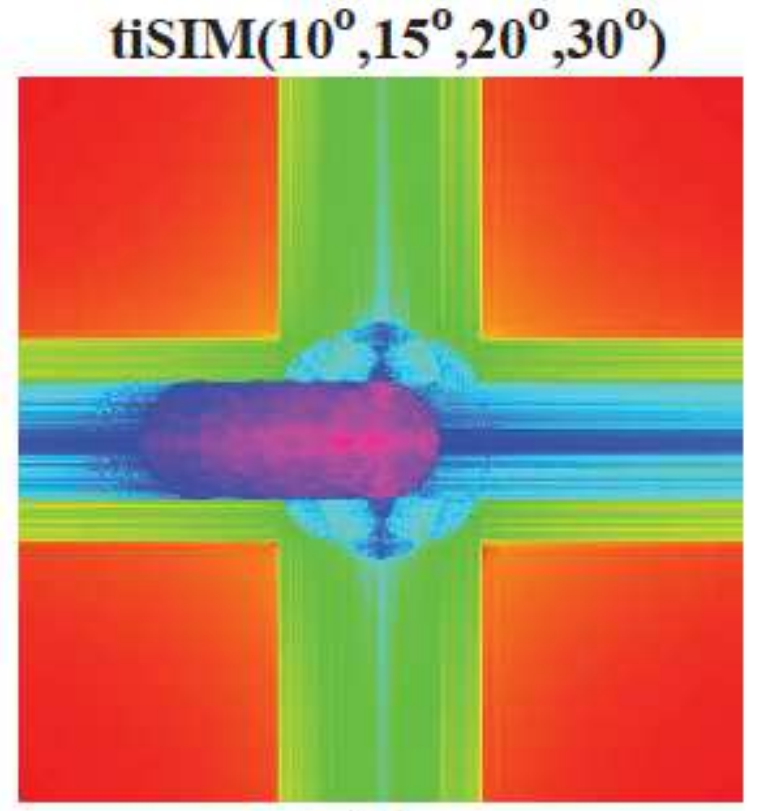

(b)

Figure 7

The frequency spectrums of the recovery images by tiSIM $\left(10^{\circ}\right)$ and tiSIM $\left(10^{\circ}, 15^{\circ}, 20^{\circ}, 30^{\circ}\right)$. Here the parts in dark circular closure curves are frequency spectrum. 


\section{$\operatorname{tiSIM}\left(\gamma_{1}, \gamma_{2}, \gamma_{3}, \ldots, \gamma_{k}\right)$}

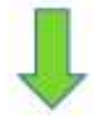

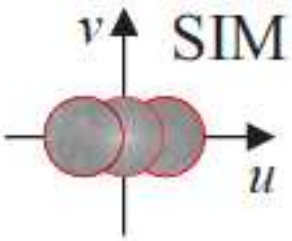

(a)

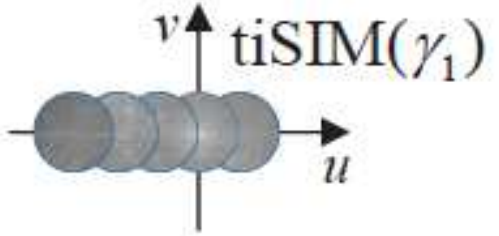

(b)

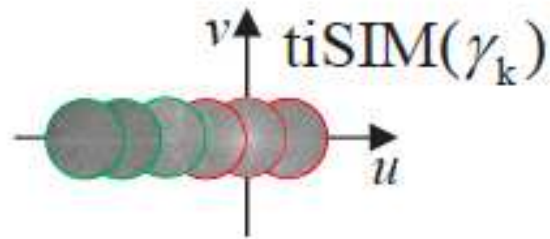

(c)

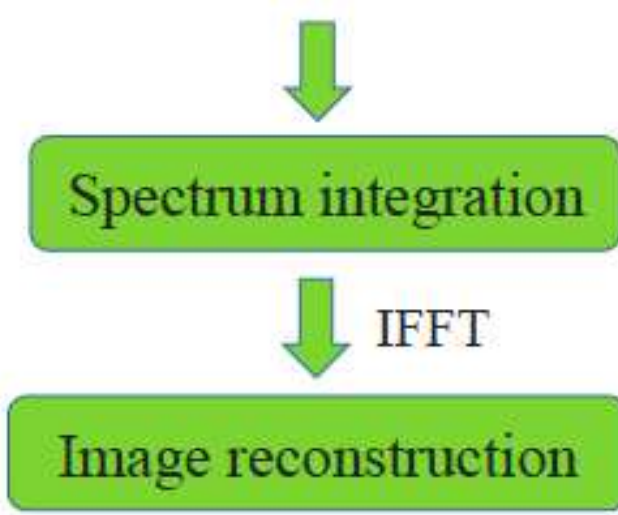

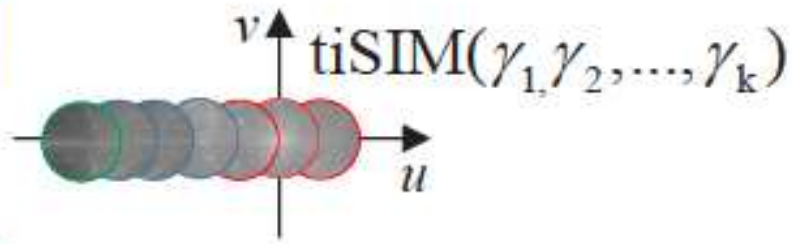

(d)

\section{Figure 8}

A flowchart of tiSIM with multi-angle illumination. (a)-(c) represent the spectrum generated by SIM, tiSIM $(\mathrm{\gamma} 1)$ and tiSIM ( $\mathrm{YK})$. (d) is an integrated spectrum. 


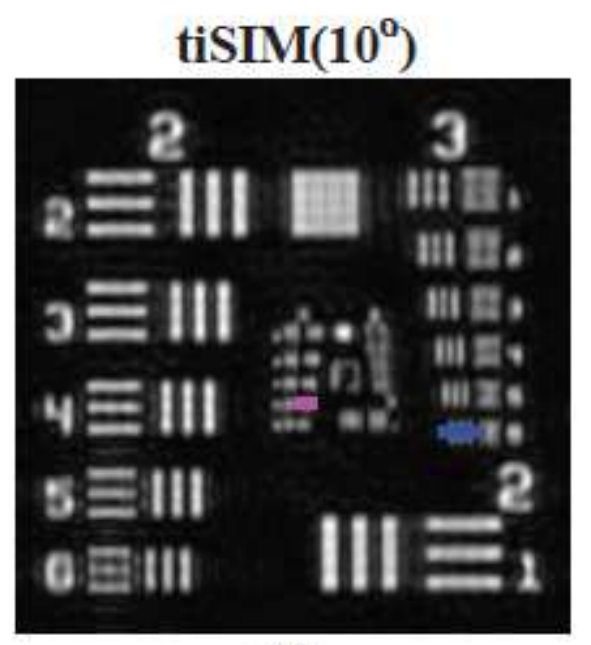

(a)

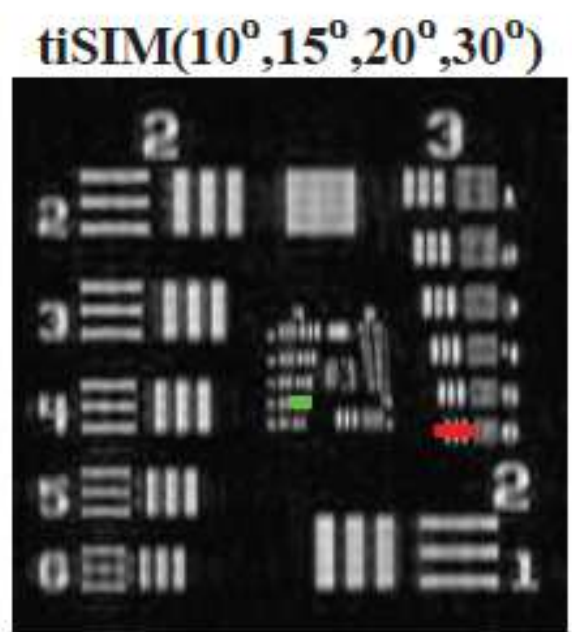

(b)

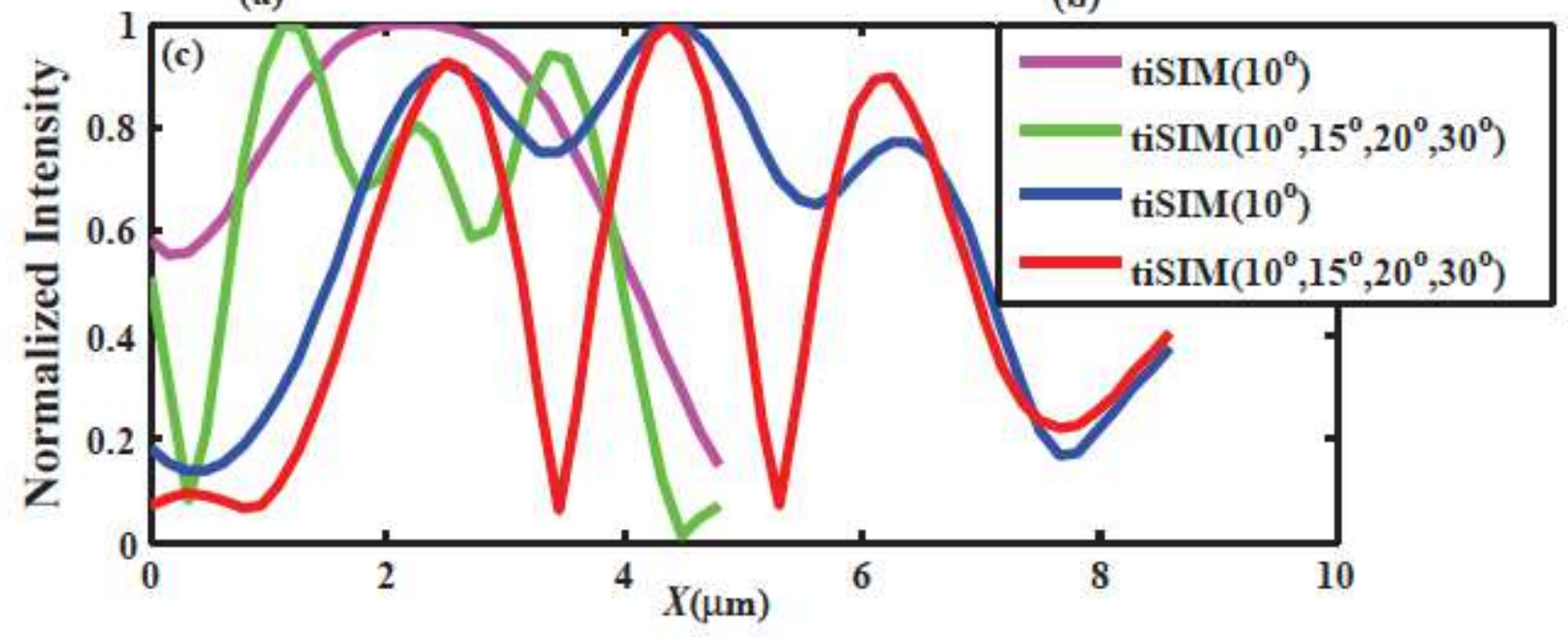

Figure 9

The reconstructed images: (a) by tiSIM $\left(10^{\circ}\right)$, (b) tiSIM $\left(10^{\circ}, 15^{\circ}, 20^{\circ}, 30^{\circ}\right)$, (c) normalized intensity profiles from specified range. 

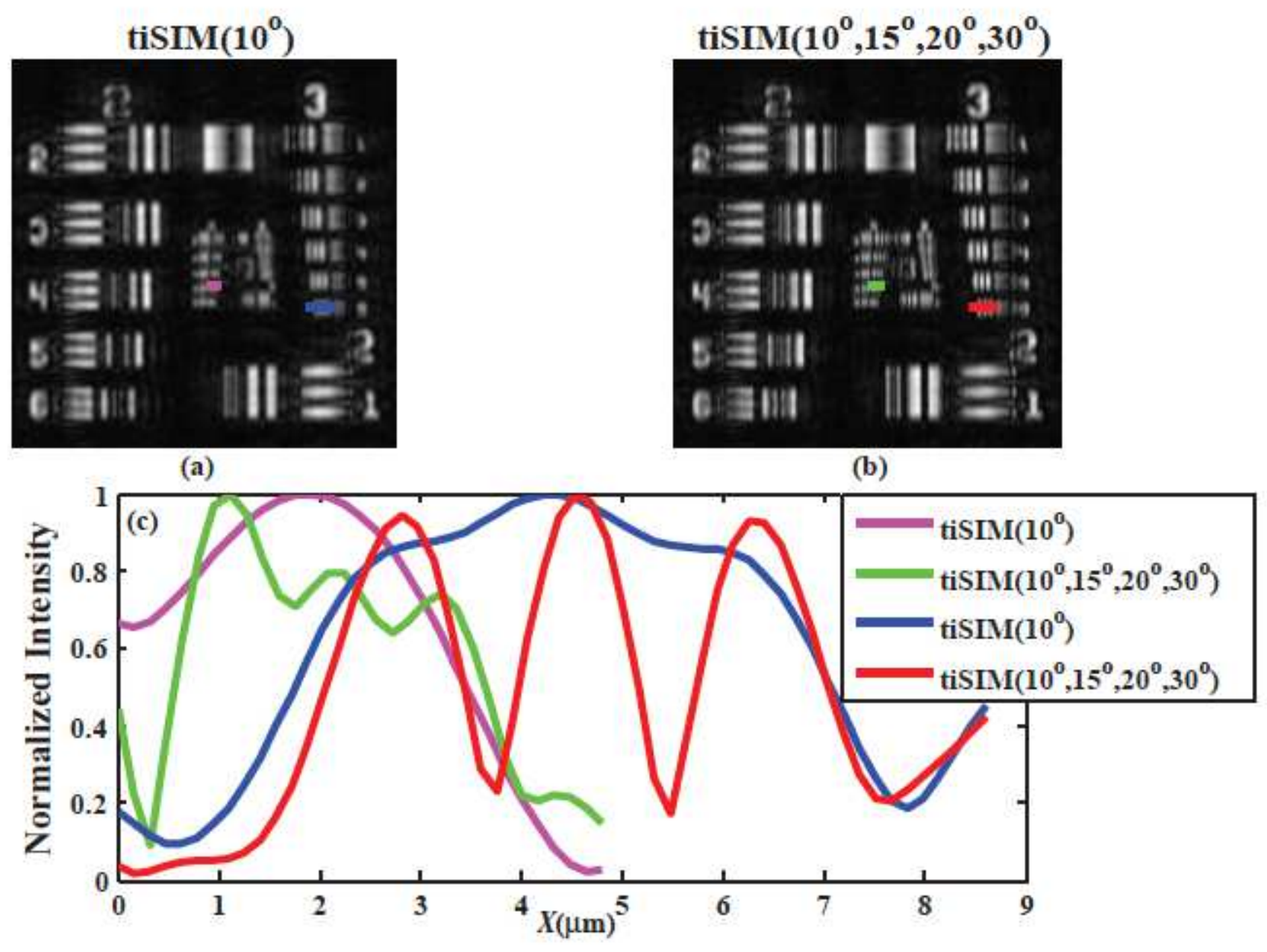

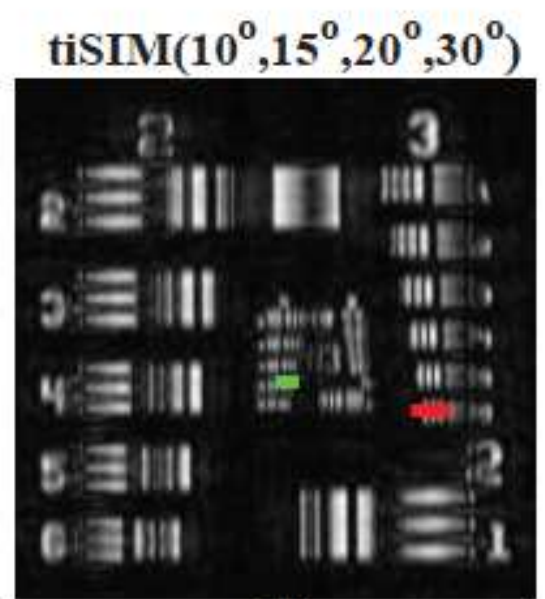

(b) 
Figure 11

Spectra and reconstructed images: (a) by WF, (b) by SIM, (c) by third-order NSIM. 